\title{
The E3 ligase Thin controls homeostatic plasticity through neurotransmitter release repression
}

\author{
Martin Baccino-Calace ${ }^{1,2}$, Katharina Schmidt ${ }^{1}$ \& Martin Müller ${ }^{1,2,3}$ \\ ${ }^{1}$ Department of Molecular Life Sciences, University of Zurich, Winterthurerstrasse \\ 190, 8057 Zurich, Switzerland \\ 2Zurich Ph.D. Program in Molecular Life Sciences, Winterthurerstrasse 190, 8057 \\ Zurich, Switzerland \\ ${ }^{3}$ Neuroscience Center Zurich, University of Zurich/ETH Zurich, Zurich, 8057 Zurich, \\ Switzerland
}

\section{ABSTRACT}

Synaptic proteins and synaptic transmission are under homeostatic control, but the relationship between these two processes remains enigmatic. Here, we systematically investigated the role of E3 ligases, key regulators of protein degradation-mediated proteostasis, in presynaptic homeostatic plasticity (PHP). An electrophysiology-based genetic screen of 157 E3 ligase-encoding genes at the Drosophila neuromuscular junction identified thin, an ortholog of human tripartite motif-containing 32 (TRIM32), a gene implicated in several neural disorders, including Autism Spectrum Disorder and schizophrenia. We demonstrate that thin functions presynaptically during rapid and sustained PHP. Presynaptic thin negatively regulates neurotransmitter release under baseline conditions by limiting the number of release-ready vesicles, independent of gross morphological defects. We provide genetic evidence that thin controls release through dysbindin, a schizophrenia-susceptibility gene required for PHP. Thin and Dysbindin localize in close proximity within presynaptic boutons, and Thin degrades Dysbindin in vitro. Thus, the E3 ligase Thin links protein degradation-dependent proteostasis of Dysbindin to homeostatic regulation of neurotransmitter release.

\section{INTRODUCTION}

Nervous system function is remarkably robust despite continuous turnover of the proteins determining neural function. Work in nervous systems of various species has established that evolutionarily conserved homeostatic singling systems maintain neural activity within adaptive ranges ${ }^{1-3}$. Chemical synapses evolved mechanisms that compensate for neural activity perturbations through homeostatic regulation of neurotransmitter release ('presynaptic homeostatic plasticity', PHP) $2,4,5$, or neurotransmitter receptors (synaptic scaling) ${ }^{6}$. Several studies have established links between homeostatic control of synaptic transmission and neural disease, such as Autism Spectrum Disorder ${ }^{7}$, schizophrenia ${ }^{8}$, or Amyotrophic Lateral Sclerosis ${ }^{9,10}$. Synaptic proteins are continuously synthesized and degraded, resulting in half-lives ranging from hours to weeks 11. The Ubiquitin-Proteasome System (UPS) is a major protein degradation pathway that controls protein homeostasis, or proteostasis. E3 
ubiquitin ligases confer specificity to the UPS by catalyzing the ubiquitination of specific target proteins, thereby regulating their function or targeting them for proteasomal degradation 12 . Synaptic proteostasis, and E3 ligases in particular, have been implicated in various neural disorders ${ }^{13}$. However, our understanding of the role of E3 ligases in the regulation of synaptic transmission is very limited. While several E3 ligases have been linked to postsynaptic forms of synaptic plasticity 14, only three E3 ligases, Scrapper ${ }^{15}$, highwire ${ }^{16}$ and Ariadne- $1{ }^{17}$ have been implicated in the regulation of presynaptic function. Moreover, a systematic investigation of E3 ligase function in the context of synaptic transmission is lacking.

PHP stabilizes synaptic efficacy in response to neurotransmitter receptor perturbation at neuromuscular junctions (NMJs) of Drosophila melanogaster ${ }^{2,4,5}$, mice ${ }^{18}$, rats ${ }^{19}$, and humans ${ }^{20}$. Furthermore, there is recent evidence for PHP in the mouse cerebellum ${ }^{21}$. The molecular mechanisms underlying PHP are best understood at the Drosophila NMJ 2, because this system is amenable to electrophysiology-based genetic screens $2,22,23$. At this synapse, pharmacological or genetic impairment of glutamate receptor activity triggers a retrograde signal that enhances presynaptic release, thereby precisely compensating for this perturbation 4,5 . PHP can be induced within minutes after pharmacological receptor impairment ${ }^{5}$. Severing the motoneuron axons forming the Drosophila NMJ in close vicinity of the synapse does not impair PHP 5, indicating that the mechanisms underlying PHP act locally at the synapse. Moreover, pharmacological inhibition of protein synthesis by cyclohexamide does not affect PHP at the Drosophila NMJ ${ }^{5}$, suggesting that de novo protein synthesis is not required for PHP. By contrast, acute or sustained disruption of the presynaptic proteasome blocks PHP ${ }^{24}$, demonstrating that presynaptic UPS-mediated proteostasis is required for PHP. Furthermore, genetic data link UPS-mediated degradation of two proteins, Dysbindin and RIM, to PHP ${ }^{24}$. Yet, it is currently unclear how the UPS controls PHP. Based on the critical role of E3 ligases in UPS function, we hypothesized an involvement of E3 ligases in PHP.

Here, we realized an electrophysiology-based genetic screen to systematically analyse the role of E3 ligases in neurotransmitter release regulation and PHP at the Drosophila NMJ. This screen discovered that the E3 ligase-encoding gene thin, an ortholog of human TRIM32 25,26, controls neurotransmitter release and PHP. We provide evidence that thin regulates the number of release-ready synaptic vesicles through dysbindin, a gene linked to PHP in Drosophila and schizophrenia in humans.

\section{RESULTS}

\section{An electrophysiology-based genetic screen identifies thin}

To systematically test the role of E3 ligases in PHP, we first generated a list of genes predicted to encode E3 ligases in $D$. melanogaster (Figure 1A). To this end, we browsed the $D$. melanogaster genome for known E3-ligase domains 27,28 . Moreover, we included homologs of predicted vertebrate E3-ligases (see Figure S1). This approach yielded 281 putative E3 ligase-encoding genes (Figure 1A), significantly higher than previously predicted for $D$. melanogaster (207 genes, $\left.{ }^{27}\right)$. To explore the relationship between the number of E3 ligase-encoding genes and genome size, we plotted the number of putative E3 ligase-encoding 
A

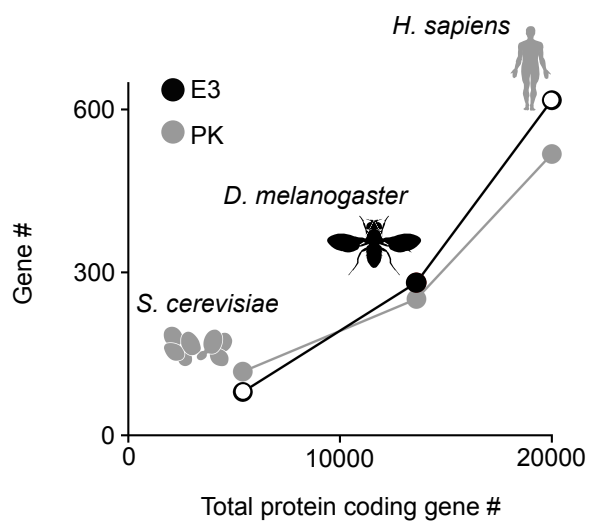

B 157 E3 ligase-encoding genes
(Presynaptic RNAi or mutants) $\rightarrow \begin{aligned} & \text { Electrophysiological } \\ & \text { PHP analysis }\end{aligned}$ EPSC WT WT + PhTX PHP mutant + PhTX

C

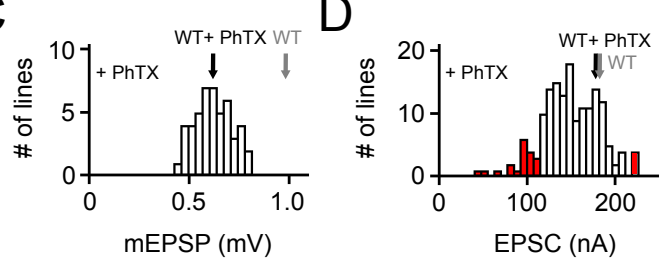

$E$

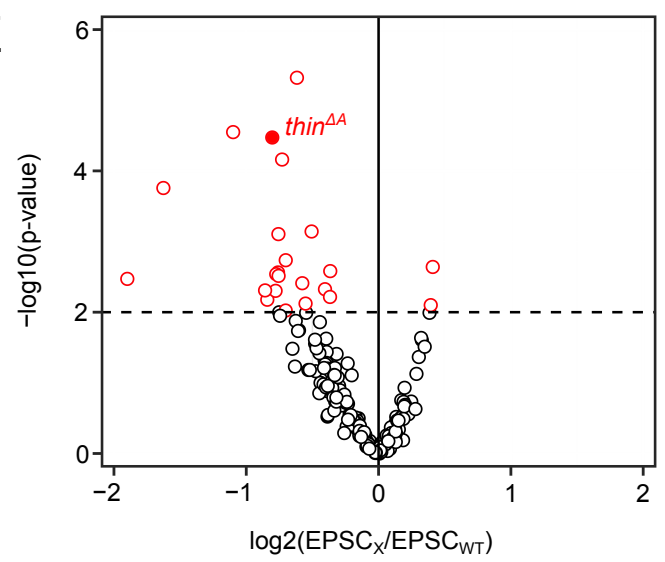

genes over the total protein-coding gene number of three species, and compared it to the relationship between protein kinase-encoding genes and genome size (Figure 1A). The relatively constant ratio between the predicted
Figure 1. An electrophysiology-based genetic screen identifies thin as a synaptic homeostasis mutant.

A) Number of putative E3 ligase-encoding genes (E3) and protein kinase-encoding genes (PK) as a function of total protein-coding gene number of $C$. cerevisiae, $D$. melanogaster, and $H$. sapiens. Note the similar relationship between E3 number, PK number and total protein-coding gene number across species. B) Top: 157 E3 ligase encoding genes and 11 associated genes (180 lines; presynaptic RNA $A_{i}$ expression, elav ${ }^{c 155}>U A S-R N A_{i}$, or mutants) were tested using two-electrode voltage clamp analysis at the Drosophila NMJ in the presence of the glutamate receptor antagonist PhTX-433 ('PhTX') to assess PHP (see Methods). Bottom: Exemplary mEPSPs and AP-evoked EPSCs recorded from WT controls, WT in the presence of PhTX ('WT + PhTX'), and a PHP mutant in the presence of PhTX ('PHP mutant + PhTX'). Note the decrease in mEPSP amplitude after PhTX treatment, indicating GluR inhibition, and the similar EPSC amplitude between WT and WT + PhTX, suggesting PHP. Small EPSC amplitudes in the presence of PhTX imply a defect in PHP or baseline synaptic transmission. C) Histogram of mean mEPSP amplitudes for each transgenic or mutant line (mean $n=4$, range 3-12; $\quad \mathrm{N}=180$ lines) following PhTX treatment. The wild-type (WT) averages under control conditions ('WT' $\mathrm{n}=16$ ) and in the presence of PhTX ('WT + PhTX', n=16) are shown as gray and black arrows, respectively. D) Histogram of mean EPSC amplitudes (as in C). The red bars indicate transgenic or mutant lines with EPSC amplitudes significantly different to the WT control in the presence of PhTX. E) Volcano plot of the ratio between the mean EPSC amplitude of a transgenic or mutant line and WT ('EPSC $C_{x} / E P S C_{W T}$ ') in the presence of PhTX ( $p$ values from one-way ANOVA with Tukey's multiple comparisons). Transgenic or mutant lines with mean EPSC amplitude changes with $p \leq 0.01$ (dashed line) are shown in red. A deletion in the gene thin (CG15105; thin $^{\triangle A}$; LaBeau-DiMenna et al., 2012) that was selected for further analysis is shown as a filled red circle. One-way ANOVA with Tukey's multiple comparisons was performed for statistical testing $(C, D, E)$.

number of E3 ligase-encoding genes and genome size across species ( 0.02-0.03; Figure 1A; $\left.{ }^{28}\right)$, suggests an evolutionarily conserved stoichiometry between E3 ligases and target proteins, similar to protein kinases (Figure 1A). 
Hence, a core mechanism of the UPS protein ubiquitination - is likely conserved in D. melanogaster.

After prioritizing for evolutionarily-conserved genes that were shown or predicted to be expressed in the nervous system (Figure S1), we investigated PHP after genetic perturbation of 157 putative E3 ligase genes and 11 associated genes (180 lines, Table S1, Figure 1B). Specifically, we recorded spontaneous mEPSPs and AP-evoked EPSCs after applying sub-saturating concentrations of the glutamate receptor (GluR) antagonist PhTX-433 (PhTX) for 10 minutes $\left(20 \mu \mathrm{M}\right.$; extracellular $\mathrm{Ca}^{2+}$ concentration, $1.5 \mathrm{mM}$ ). At WT NMJs, PhTX treatment significantly reduced mEPSP amplitude compared to untreated controls (Figure 1C, black and gray arrow), indicating GluR perturbation. By contrast, AP-evoked EPSC amplitudes were similar between PhTX-treated and untreated WT NMJs (Figure 1D, black and gray arrow). Together with a reduction in mEPSP amplitude, a similar EPSC amplitude suggests a homeostatic increase in neurotransmitter release after PhTX treatment, consistent with PHP ${ }^{5}$. PhTX also reduced mean mEPSP amplitudes in the 180 transgenic or mutant lines (either presynaptic/neural RNAi expression, elav ${ }^{c 155}$-Gal4 > UAS-RNAi; or mutations within the respective coding sequence, see Methods) compared to untreated WT controls (Figure 1C). Moreover, the mean EPSC amplitude of the majority of the tested lines did not differ significantly from the mean WT EPSC recorded at PhTXtreated NMJs (Figure 1D, white bars). The combination of a decrease in mEPSP amplitude and largely unchanged EPSC amplitude indicates that the majority of the tested lines likely display PHP. We also identified 21 transgenic or mutant lines with significantly smaller EPSC amplitudes compared to PhTX-treated WT NMJs, and two lines with increased EPSC amplitudes (Figure 1D, E, red data). These represent candidate mutations that may disrupt PHP. One of the mutant lines with significantly smaller EPSC amplitudes in the presence of PhTX was a previously described deletion of the gene thin (CG15105, thin ${ }^{\Delta A} ;{ }^{25}$ ) (Figure 1E, filled red data). This gene was selected for further analysis.

\section{Presynaptic thin promotes rapid PHP expression}

In the genetic screen, we compared synaptic transmission between a given genotype and WT controls in the presence of PhTX (Figure 1C-E). Hence, the small EPSC amplitude of thin $\Delta A$ mutants seen after PhTX application could be either due to impaired PHP, or a defect in baseline synaptic transmission. To distinguish between these possibilities, we next quantified synaptic transmission in the absence and presence of PhTX in thin ${ }^{\Delta A}$ mutants (Figure 2). Similar to WT controls, PhTX application significantly reduced mEPSC amplitude by $\sim 40 \%$ in thin $^{\triangle A}$ mutants (Figure 2A, B), suggesting similar receptor impairment. At WT synapses, EPSC amplitudes were similar in the absence and presence of PhTX (Figure 2A and C). In combination with the decrease in mEPSC amplitude (Figure 2B), PhTX incubation increased quantal content (EPSC amplitude/mEPSC amplitude) in WT (Figure 2D), indicating homeostatic release potentiation. By contrast, PhTX treatment significantly reduced EPSC amplitudes in thin ${ }^{\Delta A}$ mutants (Figure 2A, $C)$ and did not increase quantal content (Figure 2D). These data indicate that thin $\Delta A$ is required for acute PHP expression. 

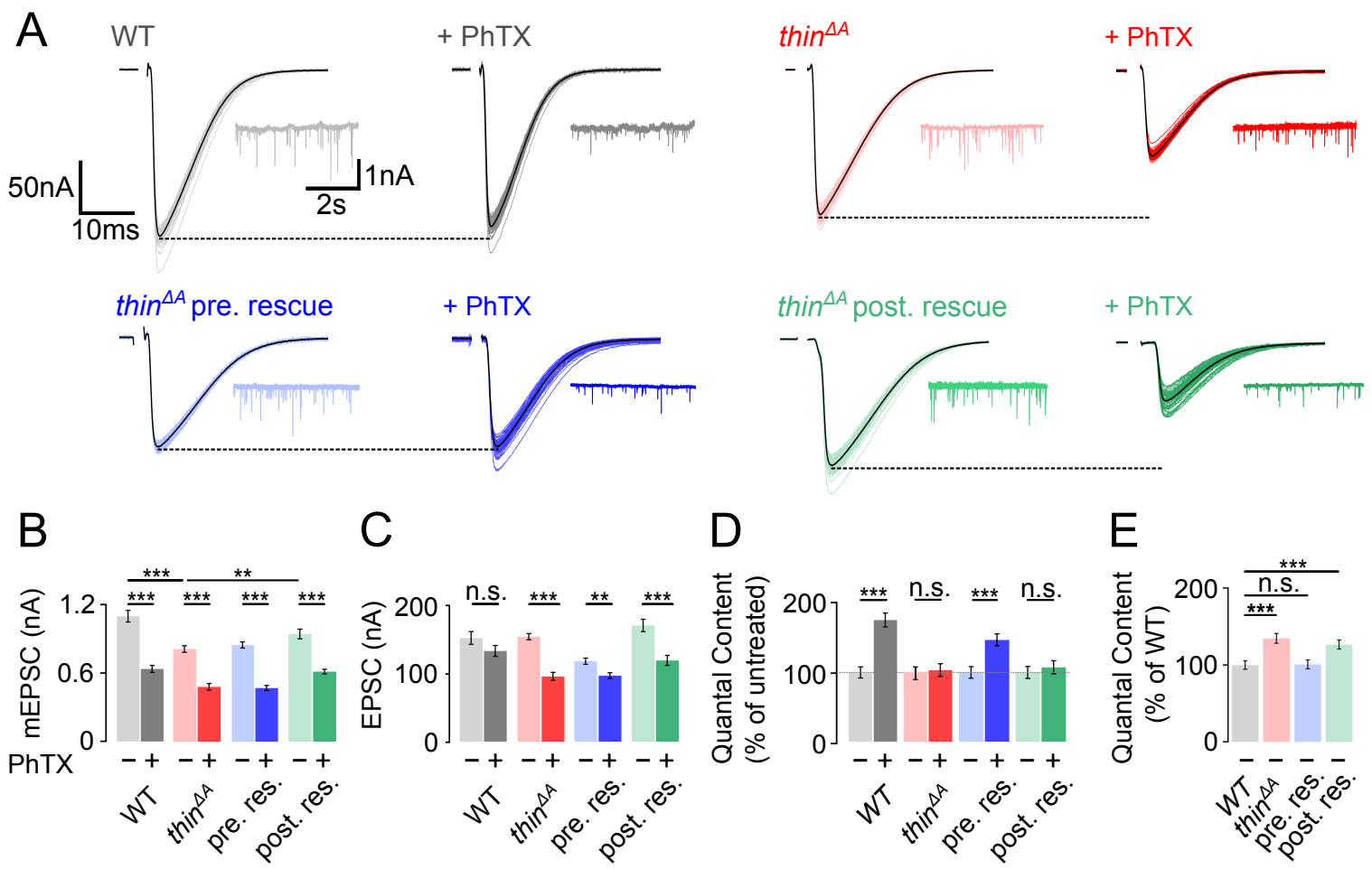

Figure 2. Homeostatic plasticity requires presynaptic thin.

A) Representative EPSCs (individual sweeps and averages are shown in light colors and black, respectively), and mEPSCs (insets) of WT (gray), thin ${ }^{\triangle A}$ mutants (red), presynaptic thin expression (elav ${ }^{C 155}$-Gal4 > UAS-thin, 'thin ${ }^{\Delta A}$ pre rescue', blue), and postsynaptic thin expression (24B-Gal4 > UAS-thin, 'thin ${ }^{\triangle A}$ post rescue', green) in the thin ${ }^{\triangle A}$ mutant background in the absence and presence of PhTX ('+ PhTX', darker colors). Stimulation artifacts were blanked for clarity. Note the decreased EPSC amplitudes at PhTX-treated thin ${ }^{\triangle A}$ mutant NMJs and thin ${ }^{\triangle A}$ post rescue NMJs, indicating impaired PHP. B - E) Mean mEPSC amplitudes (B), EPSC amplitudes (C), quantal content after PhTX treatment normalized to the respective untreated control, in the absence ('-') and presence (' + ') of PhTX (D), and baseline quantal content of the indicated genotypes in the absence ('-') of PhTX normalized to WT (E). Note that PhTX did not enhance quantal content in thin ${ }^{\triangle A}$ mutants, indicating impaired PHP. Also note the increased quantal content under baseline conditions in thin ${ }^{\triangle A}$ mutants, suggesting increased release. Both phenotypes are restored upon presynaptic thin expression in the mutant background. Mean \pm s.e.m.; $n \geq 23$ NMJs; ${ }^{*} p<0.05 ;{ }^{* *} p<0.001 ;{ }^{* * *} p<$ 0.0001 ; n.s.: not significant; two-way ANOVA followed by Tukey's post hoc test (B, C, D) and oneway ANOVA with Tukey's multiple comparisons (E).

To test if presynaptic or postsynaptic thin ${ }^{\Delta A}$ promotes PHP, we assessed PHP after presynaptic or postsynaptic expression of a thin transgene in the $\operatorname{thin}^{\triangle A}$ mutant background. PhTX treatment significantly reduced mEPSC amplitudes after neural/presynaptic (elav ${ }^{\text {c155-Gal4) }}$ or postsynaptic (24BGal4) expression of thin (UAS-thin) in thin $\triangle A$ mutants (Figure 2A, B). While EPSC amplitudes were similar in the absence and presence of PhTX after presynaptic thin expression ('presynaptic rescue' or 'pre. rescue'; Figure 2A, C, blue data), PhTX application significantly reduced EPSC amplitudes after postynaptic thin expression in the thin $^{\Delta A}$ mutant background ('postsynaptic rescue' or 'post. rescue'; Figure 2A, C, green data). Together with the decrease in mEPSC amplitude, presynaptic, but not postsynaptic thin expression significantly enhanced quantal content in the thin ${ }^{\Delta A}$ mutant background (Figure 2D). Thus, presynaptic, but not postsynaptic thin expression restores 
PHP in the thin ${ }^{\triangle A}$ mutant background, implying a presynaptic role for thin in PHP.

We also noted a decrease in mEPSC amplitude in thin $\triangle A$ mutants compared to WT in the absence of PhTX (Figure 2A, B), which is most likely due to impaired muscle architecture in thin ${ }^{\Delta A}$ mutants 25,26 . Postsynaptic, but not presynaptic thin expression, significantly increased mEPSC amplitudes towards WT levels in the thin ${ }^{\triangle A}$ mutant background (Figure $2 \mathrm{~A}, \mathrm{~B}$ ), suggesting that postsynaptic thin is required for normal MEPSC amplitude levels. Furthermore, thin $^{\Delta A}$ mutants displayed a significant increase in quantal content compared to WT under baseline conditions in the absence of PhTX (Figure 2E), which was rescued by presynaptic, but not

postsynaptic thin expression (Figure $2 \mathrm{E})$. These data are consistent with the idea that presynaptic thin represses release under baseline conditions (see Figure 4, 7). By extension, the increased release under baseline conditions in thin $\triangle A$ mutants may occlude PHP in response to receptor perturbation (see Discussion).

\section{thin promotes sustained PHP expression}

PHP is not only expressed after acute pharmacological receptor perturbation, but also upon sustained genetic receptor impairment. At the Drosophila NMJ, genetic ablation of the GluRIIA subunit in GluR/IA ${ }^{S P 16}$ mutants reduces quantal size and induces sustained PHP 4. To test if thin is required for sustained PHP expression, we
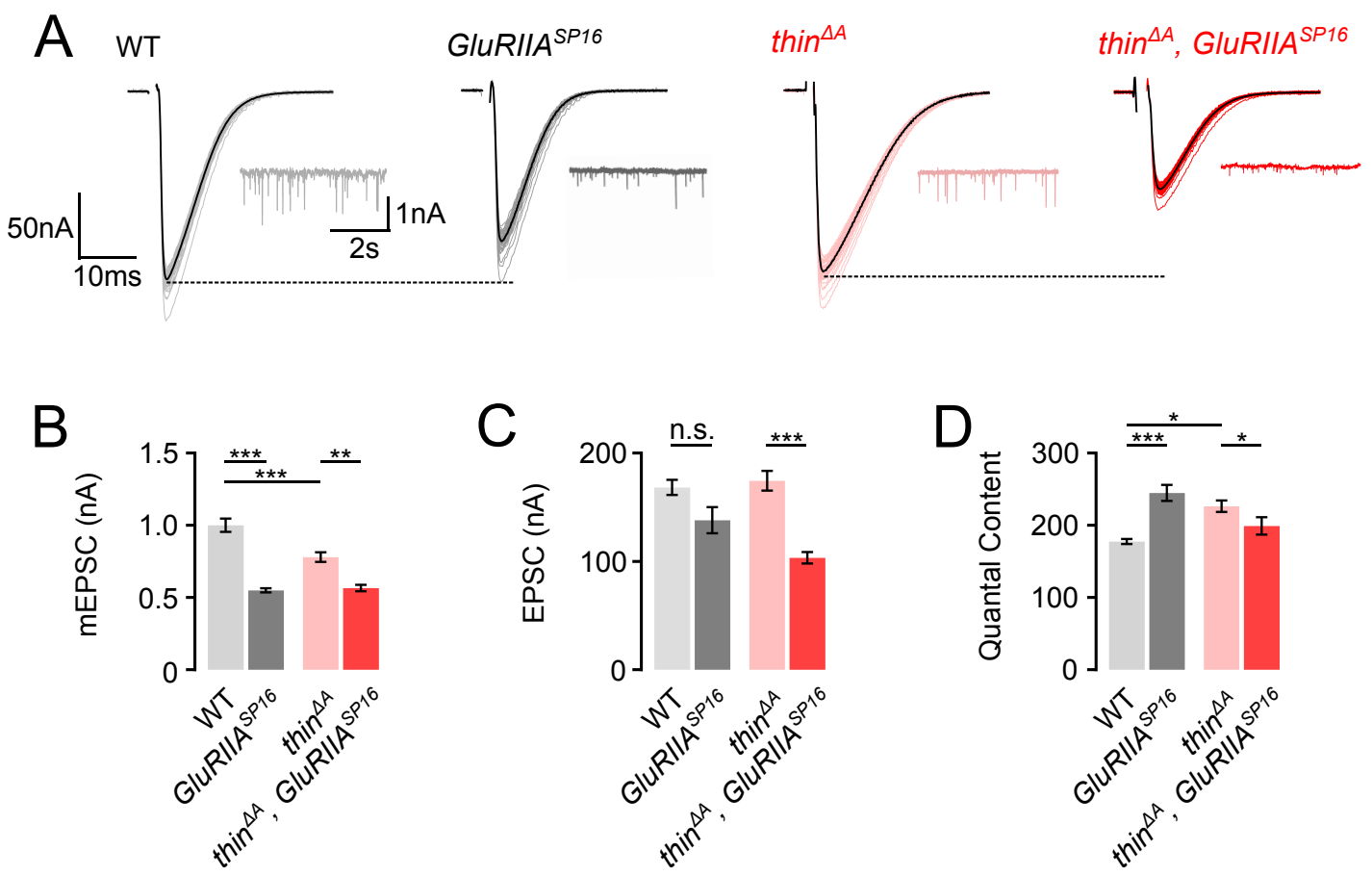

Figure 3. Sustained homeostasis is impaired in thin mutants.

A) Representative EPSCs (individual sweeps and averages are shown in light colors and black, respectively), and mEPSCs (insets) of WT (gray), GluRIIA ${ }^{S P 16}$ mutants (dark gray), thin ${ }^{\Delta A}$ mutants (red), and thin $\triangle A$, GluRIIA ${ }^{S P 16}$ double mutants (dark red). Stimulation artifacts were blanked for clarity. B - D) Mean mEPSC amplitudes (B), EPSC amplitudes (C), and quantal content (D) of the indicated genotypes. Note that there is no quantal content increase in thin ${ }^{\triangle A}$, GluRIIASP16 compared to thin ${ }^{\Delta A}$, indicating impaired PHP. Mean \pm s.e.m.; $n \geq 13$ NMJs; ${ }^{*} p<0.05 ;{ }^{* *} p<0.001 ;{ }^{* \star *} p<0.0001$; n.s.: not significant; Two-way ANOVA followed by Tukey's post hoc test. 
generated recombinant flies carrying the GluRIIA ${ }^{S P 16}$ and the thin ${ }^{\triangle A}$ mutation ('GluRIIA ${ }^{S P 16}$, thin ${ }^{\triangle A}$ '). GluRIIA $A^{S P 16}$ mutant NMJs displayed a strong decrease in mEPSC amplitude compared to WT (by $\sim 50 \%$; Figure $3 \mathrm{~A}$, B), which was accompanied by a significant increase in quantal content (Figure 3D) that restored EPSC amplitudes towards WT levels (Figure $3 \mathrm{~A}, \mathrm{C})$, in line with previous work ${ }^{4}$. By contrast, while mEPSC amplitudes were decreased by $\sim 40 \%$ in GluRIIA ${ }^{S P 16}$, thin ${ }^{\triangle A}$ double mutants with regard to thin ${ }^{\Delta A}$ mutants (Figure $3 A, B$ ), there was no increase in quantal content in GluRIIASP16, thin ${ }^{\triangle A}$ double mutants (Figure 3D), resulting in significantly smaller EPSC amplitudes than in thin ${ }^{\Delta A}$ mutants (Figure $3 \mathrm{~A}, \mathrm{C}$ ). Hence, thin is also necessary for sustained PHP expression, providing independent evidence for its role in homeostatic release regulation.

\section{thin negatively regulates release- ready vesicle number}

Having established that thin is essential for acute and sustained PHP, we next explored the role of thin in the regulation of neurotransmitter release under baseline conditions. thin ${ }^{\Delta A}$ mutants display increased neurotransmitter release in the absence of PhTX, and this increase in release is rescued by presynaptic thin expression (Figure 2).
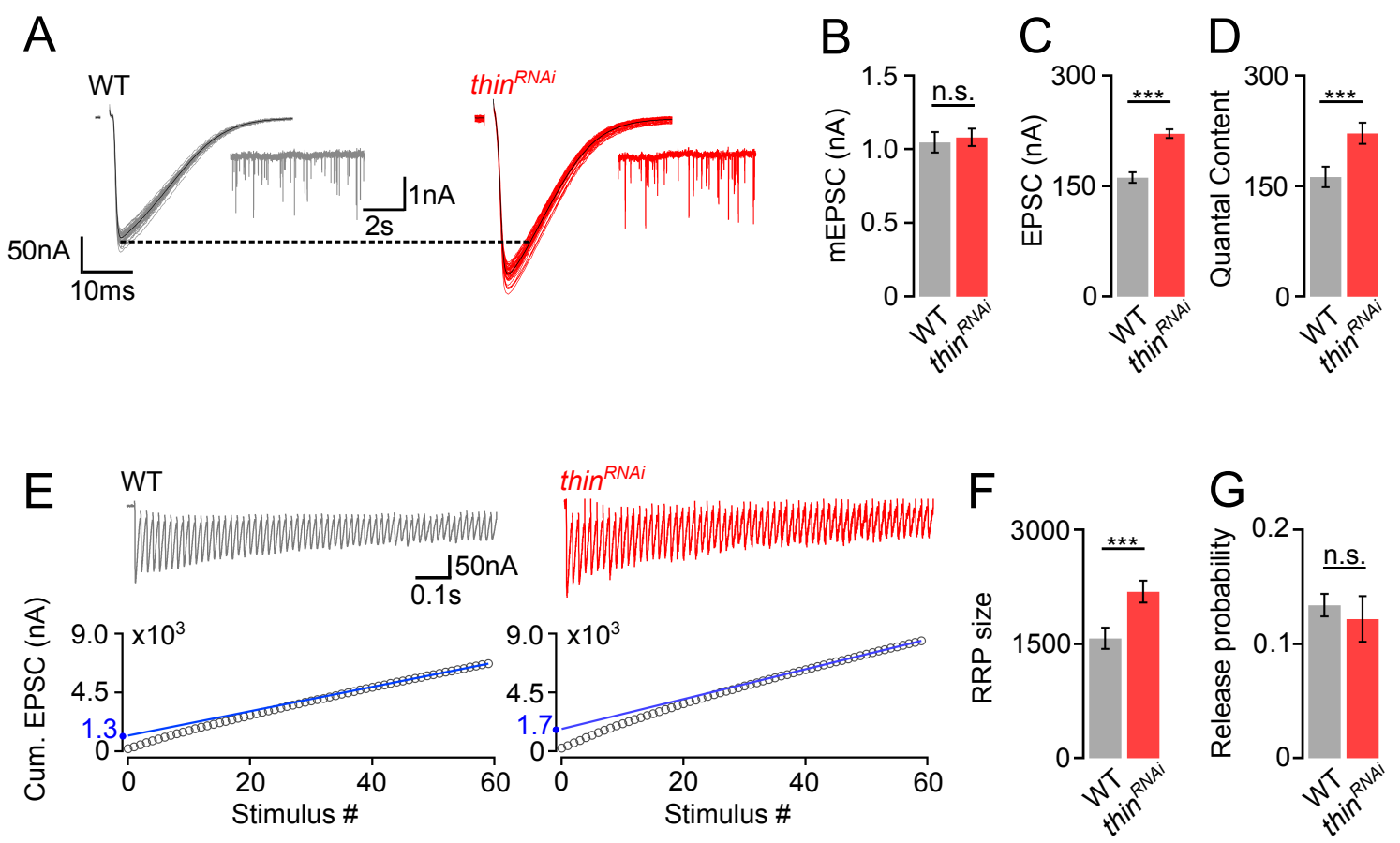

Figure 4. thin negatively regulates release-ready vesicle number.

A) Representative EPSCs (individual sweeps and averages are shown in light colors and black, respectively), and mEPSCs (insets) of WT (gray) and presynaptic thin ${ }^{\text {RNAi }}$ (elav ${ }^{155}$-Gal4 > UASthin $^{R N A i}$, red). B - D) Mean mEPSC amplitudes (B), EPSC amplitudes (C), and quantal content (D) of the indicated genotypes. E) Representative EPSC train (60 Hz, 60 stimuli, top) and cumulative EPSC amplitudes ('cum. EPSC', bottom) of WT and presynaptic thin ${ }^{R N A i}$. F, G) Mean readilyreleasable vesicle pool (RRP) size (cum. EPSC/mEPSC) (F), and release probability (EPSC/cum. EPSC) (G) of the indicated genotypes. Note the increase in EPSC amplitude and RRP size in presynaptic thin ${ }^{R N A i}$. Mean \pm s.e.m.; $n \geq 22$ NMJs; ${ }^{*} p<0.05$; ${ }^{* *} p<0.001 ;{ }^{* * *} p<0.0001$; ns: not significant; Student's $t$-test. 
To elucidate the mechanisms through which thin negatively modulatesrelease, we probed the size of the readilyreleasable pool of synaptic vesicles (RRP) and neurotransmitter release probability $\left(p_{r}\right)$ after presynaptic thin perturbation (Figure 4). As the decreased mEPSC amplitude in thin ${ }^{\triangle A}$ mutants may confound conclusions regarding presynaptic thin function (Figure 2), we focused our further analyses on the effects of presynaptic thin $^{R N A i}$ expression (Figure 4). Presynaptic thin ${ }^{R N A i}$ expression $\left(\right.$ elav $^{\text {155-Gal4 }}>$ UAS-thin ${ }^{\text {RNAi }}$ ) significantly increased EPSC amplitudes (Figure 4A, C), with no significant effects on mEPSC amplitudes compared to controls (elavc155_Gal4/+; Figure 4A, B), suggesting that presynaptic thin represses release, consistent with the data obtained from thin $^{\Delta A}$ mutants (Figure 2).

Next, we estimated RRP size using cumulative EPSC amplitude analysis during high-frequency stimulation (60 Hz; ${ }^{29,30}$ ) (Figure 4E). This analysis revealed a significantly larger RRP size upon presynaptic thin ${ }^{R N A i}$ expression compared to controls (Figure 4E, F), implying that presynaptic thin negatively regulates RRP size. We then estimated $p_{r}$ based on the ratio between the first EPSC amplitude of the stimulus train and the cumulative EPSC amplitude and observed no significant $p_{r}$ differences between thin ${ }^{R N A i}$ and controls (Figure 4G). Thus, thin represses release by limiting the number of release-ready synaptic vesicles with largely unchanged $p_{r}$.

\section{Altered NMJ development unlikely causes PHP defect in thin mutants}

The PHP defect and the release enhancement under baseline conditions after presynaptic thin perturbation may arise from impaired synaptic development. To test this possibility, we investigated NMJ morphology in thin mutants (Figure 5). We confined the morphological analysis to NMJs lacking thin presynaptically (thin $\triangle A$; 24BGal4>UAS-thin; henceforth called 'presynaptic thin ${ }^{\triangle A}$ mutant'; Figure $5 \mathrm{~B}$ ), because ubiquitous loss of thin impairs muscle development 25,26. Immunostainings with an antibody detecting neuronal membrane (antihorseradish peroxidase, 'HRP'; ${ }^{31}$ ) revealed a slight, but significant increase in HRP area in presynaptic thin ${ }^{\Delta A}$ mutants compared to WT (Figure $5 \mathrm{C}$ ), indicating a slight increase in NMJ size. Analysis of the active zone marker Bruchpilot (anti-Bruchpilot, 'Brp'; ${ }^{32}$ ) uncovered a significant increase in Brp puncta number per NMJ (Figure 5D), a slight increase in Brp density (Figure $5 \mathrm{E})$, as well as a decrease in Brp intensity (Figure $5 \mathrm{~F}$ ) in presynaptic thin $^{\Delta A}$ mutants. In principle, these morphological changes could be related to the PHP defect or release enhancement seen in thin ${ }^{\triangle A}$ mutants. However, postsynaptic thin expression in WT induced similar morphological alterations compared to thin overexpression in $\operatorname{thin}^{\Delta A}$ mutants (presynaptic thin $\triangle A$ mutants) (Figure S2), but neither impaired PHP nor enhanced release (Figure S2). These data suggest that the morphological changes seen in presynaptic thin $\Delta A$ mutants are caused by postsynaptic thin expression rather than the thin $\triangle A$ mutation, and that these morphological alterations do not block PHP or enhance release. Together, we conclude that the PHP defect and the increase in baseline synaptic transmission in thin mutants are unlikely caused by major changes in NMJ development. 

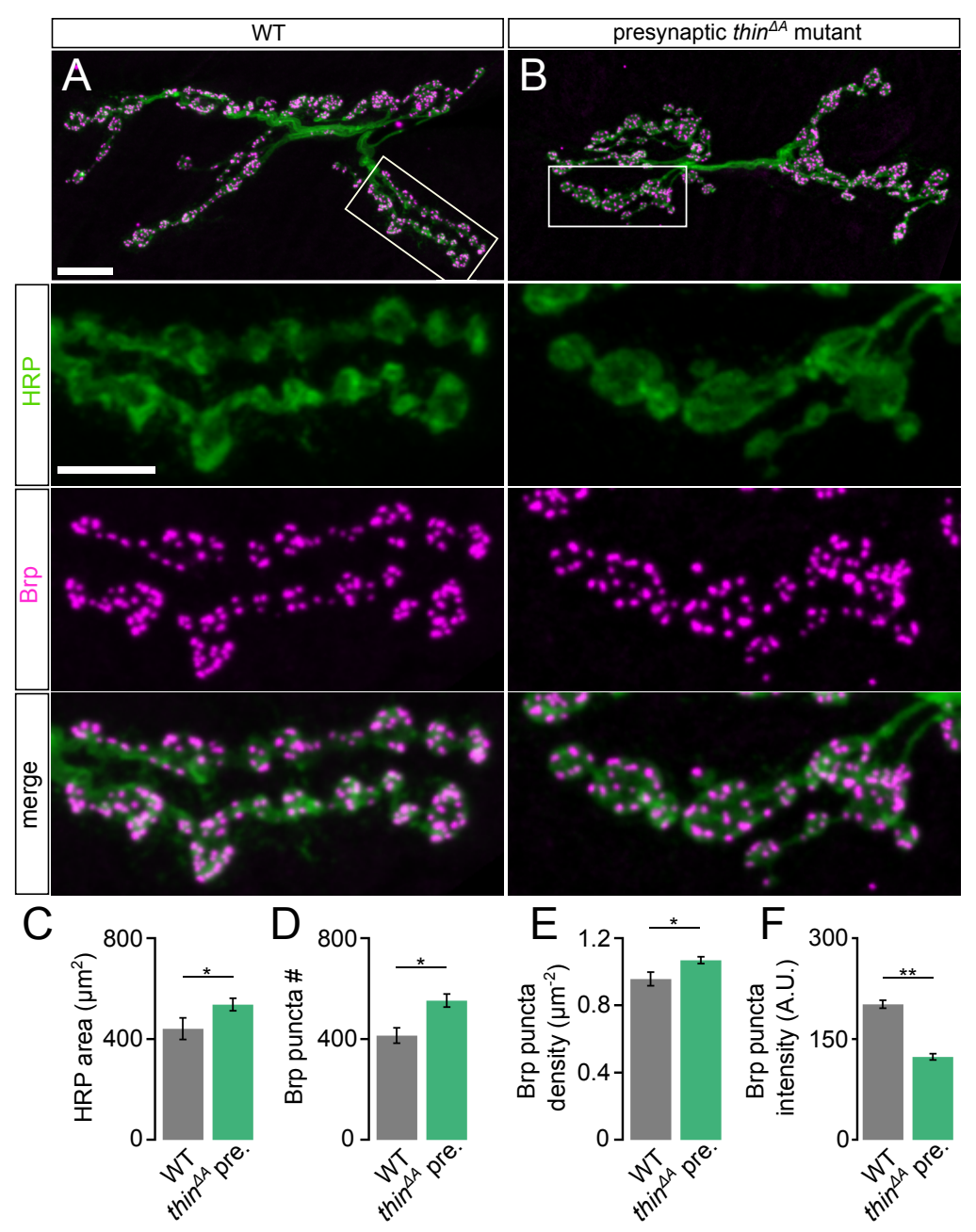

Figure 5. Altered NMJ development unlikely causes PHP defect in thin mutants.

A) Maximum intensity projection of a WT NMJ, and B) an NMJ lacking thin presynaptically (thin ${ }^{\triangle A} ; \quad 24 B-G a l 4>U A S-t h i n$ or 'thin ${ }^{\triangle A}$ pre.' stained against the Drosophila neuronal membrane marker anti-HRP ('HRP') and the active-zone marker Bruchpilot ('Brp'); scale bar, overview, 10 $\mu \mathrm{m}$; inset, $5 \mu \mathrm{m}$. C - F) Mean HRP area ('HRP area', C), Brp puncta number per NMJ ('Brp puncta \#', D), Brp puncta number/HRP area per NMJ ('Brp puncta density', E), Brp puncta fluorescence intensity ('Brp puncta intensity', F). Loss of presynaptic thin induces a slight, but significant increase in the total number of brp puncta. Mean \pm s.e.m.; $n \geq 10$ NMJs; " $p<0.05$; ${ }^{* *} p<0.001 ;{ }^{* * *} p<0.0001$; ns: not significant; Student's t-test.
Thin localizes in close proximity to Dysbindin and promotes Dysbindin degradation

Trim32, Thin's human ortholog, ubiquitinates Dysbindin and targets it for degradation ${ }^{33}$. dysbindin, in turn, is required for PHP at the Drosophila NMJ 22 , and genetic evidence suggests that the UPS controls Dysbindin under baseline conditions and during PHP ${ }^{24}$. We therefore explored the relationship between Thin and Dysbindin. First, we investigated the localization of Thin in relation to Dysbindin within synaptic boutons (Figure 6A). Previous studies suggest very low endogenous Dysbindin levels that preclude direct immunohistochemical analysis at the Drosophila NMJ 22,24. However, presynaptic expression of a fluorescently-tagged transgene revealed that Dysbindin localizes in close proximity to synaptic vesicle markers ${ }^{22}$ (Figure S3). The localization of fluorescently-tagged Dysbindin likely overlaps with the one of endogenous Dysbindin, as its presynaptic expression rescues the PHP defect in dysbindin mutants ${ }^{22}$. The strong anti-Thin staining of Drosophila muscles makes it difficult to distinguish between presynaptic and postsynaptic Thin ${ }^{25}$. This prompted us to analyse the localization of fluorescently-tagged Thin, which we expressed presynaptically (elav ${ }^{\mathrm{C} 155}$-Gal4 $>$ UASthin ${ }^{\text {mcherry }}$. Presynaptic Thin ${ }^{\text {mcherry }}$ partially overlapped with fluorescentlytagged Dysbindin at confocal resolution (elavC155-Gal4 > UAS-dysb ${ }^{\text {venus; }}$ Figure $6 A, B)$. The localization of fluorescentlytagged Thin also likely overlaps with endogenous Thin, because presynaptic thin expression restores PHP and 


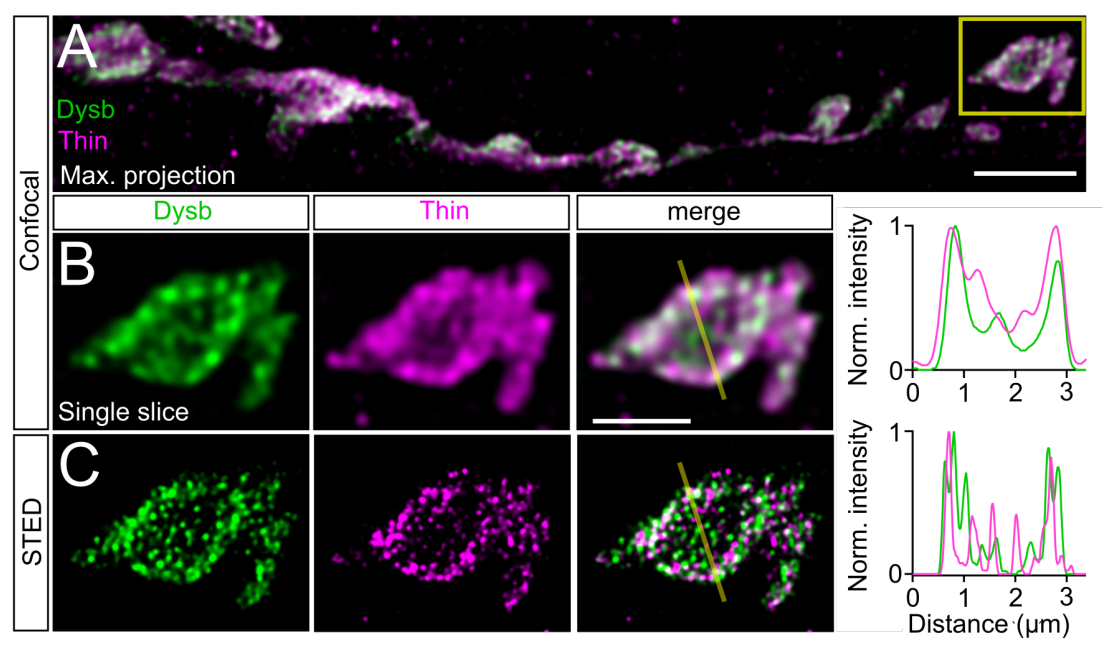

\section{Drosophila S2 cells}
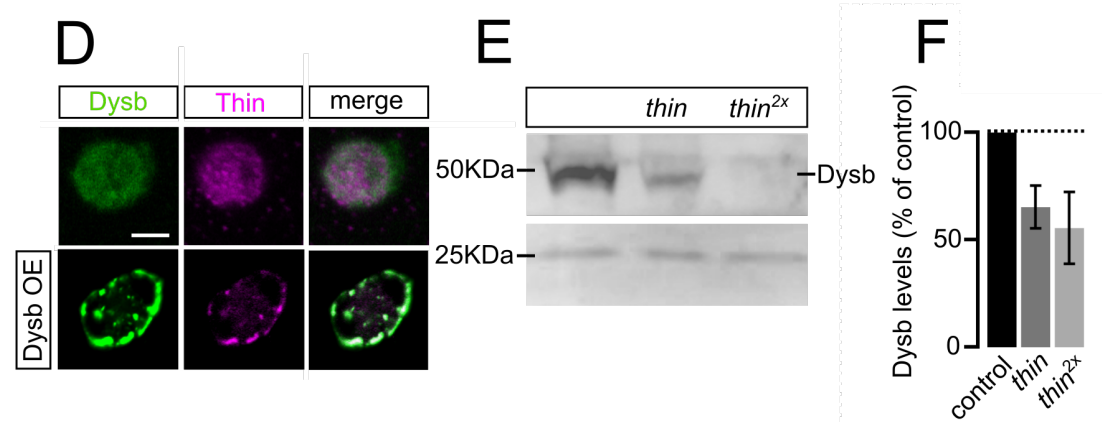

Figure 6. Thin localizes in close proximity to Dysbindin and promotes Dysbindin degradation.

A) Confocal maximum intensity projection of a representative NMJ branch (muscle 6-7) after presynaptic co-expression (elavc155_Gal4) of venus-tagged Dysbindin (UAS-dysb venus, 'Dysb', green) and mCherry-tagged Thin (UAS-thin ${ }^{\text {mcherry }}$,'Thin', magenta). B) Single plane of the synaptic bouton highlighted by the yellow square in (A) with corresponding line profile (right). The yellow line demarks the location of the line profile. C) gSTED image of the synaptic bouton shown in (B) with corresponding line profile (right). Note the partial overlap between Thin and Dysbindin at confocal and STED resolution. D) Confocal images (single planes) of Drosophila S2 cells stained with antiDysbindin (green) and anti-Thin (magenta) under control conditions (top row) and after dysbindin overexpression (UAS-dysb venus, bottom row). Note the concomitant redistribution of Dysbindin and Thin upon dysbindin overexpression. E) Representative Western blot of S2 cells transfected with $U A S-d y s b^{\text {venus }}$ and different levels of UAS-thin. F) Quantification of $(E, n=5)$. Note the decrease in Thin levels upon dysbindin overexpression. Scale bar (A: $5 \mu \mathrm{m}),(B, C: 2 \mu \mathrm{m}),(\mathrm{D}: 5 \mu \mathrm{m})$.

synaptic transmission in thin mutants (Figure 2). As indicated by the line profile across a bouton (Figure 6B), Dysbindin and Thin fluorescence intensity increased toward the bouton periphery (Figure 6B), similar to synaptic vesicle markers, such as synapsin (Figure S3B). At STED resolution, fluorescently-tagged Thin and Dysbindin appeared as distinct spots that partially overlapped (Figure $6 \mathrm{C})$. Based on the close proximity between Dysbindin and synaptic vesicle markers ${ }^{22}$, these data indicate that a fraction of Thin localizes in the close vicinity of Dysbindin and synaptic vesicles.

Previous work in cultured human cells showed that Dysbindin ubiquitination by Thin's human ortholog 
Trim32 induces Dysbindin degradation 33. To test if this function is conserved in Drosophila, we used cultured Drosophila S2 cells. First, we probed the relationship between Thin and Dysbindin localization. Interestingly, while anti-Thin fluorescence was homogenously distributed within S2 cells under control conditions (Figure 6D, top), dysbindin (dys benus) overexpression led to a redistribution of anti-Thin fluorescence into clusters that localized in close proximity to Dysbindin clusters (Figure 6D, bottom), suggesting a possible interaction between Thin and Dysbindin.

Next, we assessed whether Thin expression affects Dysbindin abundance in S2 cells by Western blot analysis. We observed a decrease in Dysb ${ }^{\text {venus }}$ levels upon co-expression of increasing Thin ${ }^{\text {mcherry }}$ levels (Figure 6E, $F)$. Together, these data are consistent with the idea that Thin acts as an E3 ligase for Dysbindin in Drosophila, similar to Trim32 in humans ${ }^{33}$.

\section{thin represses release through dysbindin}

We next explored a possible genetic interaction between thin and dysbindin in the context of synaptic physiology. As thin and dysbindin mutants alone disrupt PHP, the analysis of double mutants would not be informative. We therefore investigated baseline synaptic transmission after presynaptic thin ${ }^{R N A i}$ expression in the dysbindin mutant background (Figure 7). Neither presynaptic thin ${ }^{R N A i}$ expression $\left(\right.$ elav $^{\text {c155-Gal4 > UAS-thin }}{ }^{\text {RNAi }}$ ) in the WT background, nor in the $d y s b^{1}$ mutant background affected mEPSC amplitude (Figure 7A, B). While presynaptic thin ${ }^{R N A i}$ expression enhanced EPSC amplitude and quantal content in the WT background (Figure 7C, D; see also Figure 4), presynaptic thin ${ }^{R N A i}$ expression did not change EPSC amplitude (Figure 7C) or quantal content (Figure 7D) in the $d y s b^{1}$ mutant background. These data provide genetic evidence that thin negatively controls release through dysbindin (Figure 7E).

\section{DISCUSSION}

Employing an electrophysiology-based genetic screen targeting 157 E3 ligaseencoding genes at the Drosophila NMJ, we discovered that a mutation in the E3 ligase-encoding gene thin disrupts acute and sustained PHP. Presynaptic loss of thin led to increased release and RRP size, largely independent of gross synaptic morphological changes. Thin and Dysbindin localize in close proximity within synaptic boutons, and biochemical evidence suggests that Thin degrades Dysbindin in vitro. Finally, presynaptic thin perturbation did not enhance release in the dysbindin mutant background, providing genetic evidence that thin represses release through dysbindin.

As thin and dysbindin are

required for $\mathrm{PHP}$, these data are consistent with a model in which thin controls neurotransmitter release during PHP and under baseline conditions through dysbindin (Figure 7E, F).

Our study represents the first systematic investigation of E3 ligase function in the context of synaptic transmission. A considerable fraction of lines tested (11\%) displayed a decrease in EPSC amplitude after PhTX treatment (Figure 1C-E). These E3 ligase-encoding genes may either be required for PHP and/or baseline synaptic transmission. Previous PHP screens in the same system identified PHP mutants with a success rate of $\sim 3 \%{ }^{22,23}$. Thus, our data indicate that E3 ligase function either plays a special role in PHP and/or baseline synaptic transmission. As more transgenic or 

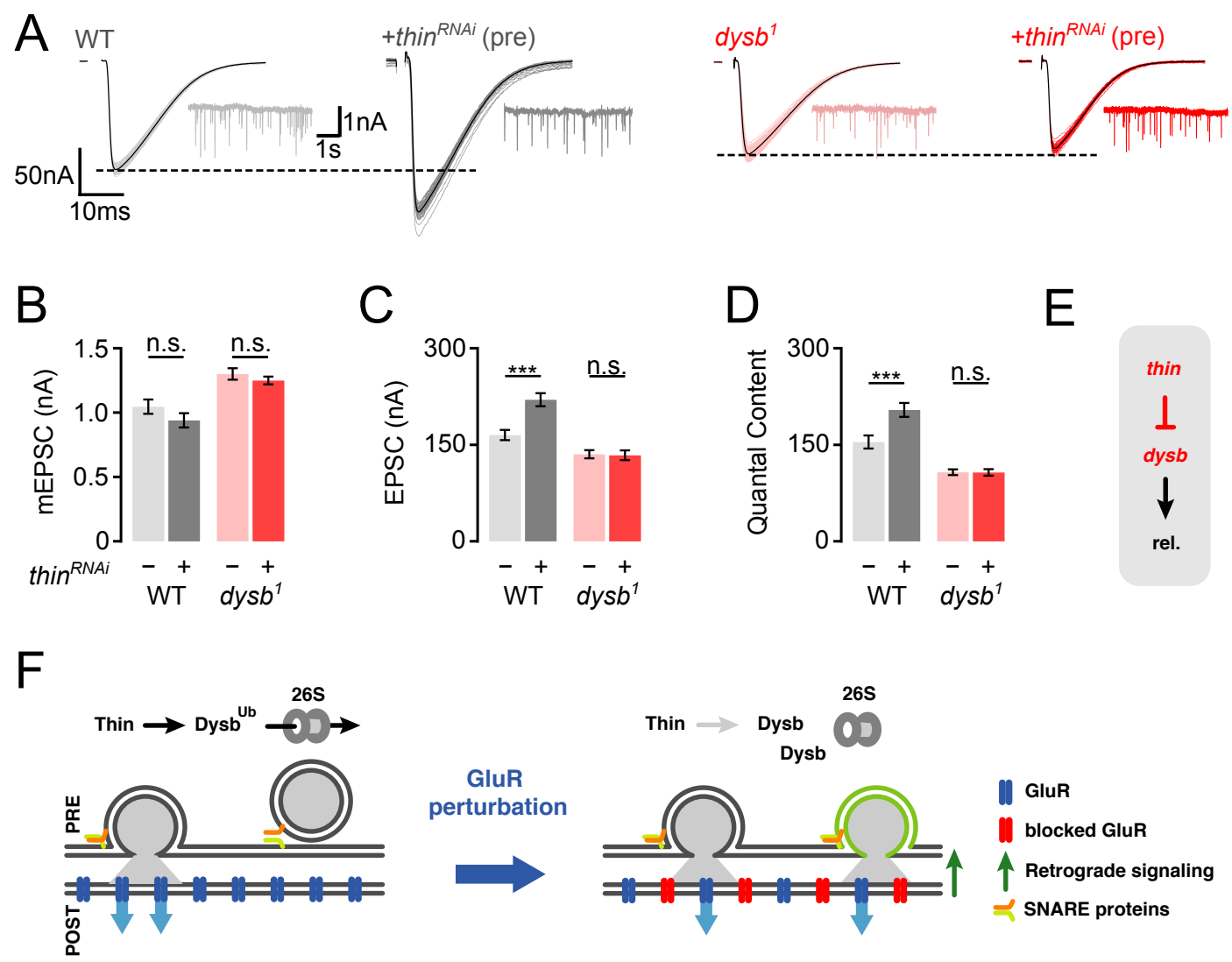

Figure 7. thin represses release through dysbindin.

A) Representative EPSCs (individual sweeps and averages are shown in light colors and black, respectively), and mEPSCs (insets) of WT (gray) and presynaptic thin ${ }^{R N A i}$ (elav ${ }^{C 155}-$ Gal4 > UASthin ${ }^{R N A i}$, dark gray), dysh ${ }^{1}$ mutants (light red), and presynaptic thin ${ }^{R N A i}$ in the dysb ${ }^{1}$ mutant background (elav ${ }^{C 15}-$ Gal4 > UAS- thin RNAi, dark red) . B - D) Mean mEPSC amplitudes (B), EPSC amplitudes (C), and quantal content (D) of the indicated genotypes. Note that presynaptic thin ${ }^{R N A i}$ expression increases EPSC amplitude and quantal content in WT, but not in $d y s b^{1}$ mutants. Mean \pm s.e.m.; $n \geq 13$ cells; ${ }^{*} p<0.05 ;{ }^{* *} p<0.001$; ${ }^{* *} p<0.0001$; ns: not significant; two-way ANOVA followed by Tukey's post hoc test. E) Working model: Our genetic data support a model in which thin controls neurotransmitter release ('rel.') through negative regulation of dysbindin. F) Emerging model: Left: Under baseline conditions, Thin ubiquitinates ('Ub') Dysbindin ('Dysb') and targets it for degradation by the $26 \mathrm{~S}$ proteasome ('26S'). Right: GluR perturbation (red GluRs) induces retrograde PHP signaling (green arrow), which decreases Thin-dependent Dysb degradation through an unknown pathway, thereby increasing Dysb levels and presynaptic release (green synaptic vesicle). Based on previous data, Dysbindin likely increases release by interacting with the SNARE complex through snapin and SNAP-25 (see Discussion).

mutant lines exhibited a decrease in synaptic transmission, we conclude that the net effect of E3 ligases is to promote synaptic transmission at the Drosophila NMJ. Given the evolutionary conservation of most E3 ligases tested in this study (Figure 1, Table S1), the results of our screen likely allow predicting the role of the tested E3 ligases in neurotransmitter release regulation in other systems.
Previous studies linked E3 ligases to synaptic development and synaptic function at the Drosophila NMJ 34,35. For instance, the E3 ligase highwire (hiw) restrains synaptic growth and promotes evoked synaptic transmission at the Drosophila NMJ ${ }^{34}$. Similarly, the deubiquitinating protease fat facets represses synaptic growth and enhances synaptic transmission ${ }^{36}$. Although different molecular pathways 
have been implicated in hiw-dependent regulation of synaptic growth and function ${ }^{16}$, it is generally difficult to disentangle effects on synaptic morphology from synaptic function. thin and its mammalian ortholog Trim32 are required for maintaining the cytoarchitecture of muscle cells $25,37,38$. Hence, the changes in synaptic transmission described in the present study may be a secondary consequence of impaired muscle structure. However, presynaptic thin expression in the thin mutant background restored synaptic function under baseline conditions and during homeostatic plasticity (Figure 2). Conversely, while postsynaptic thin expression largely rescued the defects in muscle morphology in thin mutants, the defects in synaptic function persisted. These genetic data suggest that the impairment of baseline synaptic transmission and homeostatic plasticity in thin mutants is unlikely caused by muscular dystrophy. We also noted a slight increase in Brp number at presynaptic thin mutant NMJs (Figure $5)$, indicating increased active-zone number. In principle, this increase in active-zone number may underlie the increase in neurotransmitter release after presynaptic loss of thin. However, postsynaptic thin overexpression increased Brp number in WT, but did neither affect baseline synaptic transmission, nor PHP (Figure S2). Hence, our results suggest that presynaptic thin regulates neurotransmitter release under baseline conditions and during homeostatic plasticity largely independent of changes in synaptic morphology.

We revealed that presynaptic thin perturbation results in enhanced neurotransmitter release (Figure 2, 4, 7 ), indicating that the E3 ligase Thin represses neurotransmitter release under baseline conditions. Notably, there are just a few molecules that have been implicated in repressing neurotransmitter release, such as the SNARE-interacting protein tomosyn ${ }^{39,40}$, or the RhoGAP crossveinless-c ${ }^{41}$. How could the E3 ligase Thin oppose neurotransmitter release? We discovered that $d y$ sbindin is required for the increase in release induced by presynaptic thin perturbation (Figure 7). Moreover, we revealed that Thin localizes in close proximity to Dysbindin in synaptic boutons (Figure 6), and that Thin degrades Dysbindin in vitro (Figure 6), similar to its mammalian ortholog Trim32 ${ }^{33}$. At the Drosophila NMJ, 26Sproteasomes are transported to presynaptic boutons ${ }^{42}$, where they degrade proteins on the minute time scale 24,43 . Previous genetic data suggest a positive correlation between Dysbindin levels and neurotransmitter release ${ }^{22,24}$, and there is genetic evidence for rapid, UPS-dependent degradation of Dysbindin at the Drosophila NMJ ${ }^{24}$. In combination with these previous observations, our data are consistent with the idea that Thin opposes release by acting on Dysbindin. Although the low abundance of endogenous Dysbindin at the Drosophila NMJ precludes direct analysis of Dysbindin levels ${ }^{22}$, we speculate that Thin decreases Dysbindin abundance by targeting it for degradation. Alternatively, Thin may modulate Dysbindin function through mono-ubiquitination. Genetic data suggest that Dysbindin interacts with the SNARE protein SNAP25 through Snapin 44. Hence, Thin-dependent regulation of Dysbindin may modulate release via Dysbindin's interaction with the SNARE complex.

Our study revealed a crucial role for thin in PHP. How does the increase in neurotransmitter release in thin mutants under baseline conditions relate to the PHP defect? The relative increase in release during PHP of WT synapses exceeds the increase in 
release in thin mutants under baseline conditions. Thus, although we cannot exclude that PHP is solely occluded by enhanced baseline release in thin mutants, we consider this scenario unlikely. PHP is blocked by acute pharmacological, or prolonged genetic proteasome perturbation at Drosophila NMJ ${ }^{24}$. Moreover, PHP at this synapse requires dysbindin ${ }^{22}$, and genetic data suggest UPS-dependent control of a Dysbindin-sensitive vesicle pool during $\mathrm{PHP}{ }^{24}$. Based on our finding that thin is required for acute and sustained PHP expression (Figures 2 and 3 ), and the links between thin und dysbindin in the context of release modulation outlined above, we propose a model in which Thin-dependent ubiquitination of Dysbindin is decreased during PHP (Figure 7F). Given the positive correlation between Dysbindin levels and release 24,44 , the resulting increase in Dysbindin abundance would potentiate release. Further work is needed to test how Thin is regulated during PHP. Thin is the first E3 ubiquitin ligase linked to homeostatic regulation of neurotransmitter release. Interestingly, a recent study revealed a postsynaptic role for Insomniac, a putative adaptor of the Cullin-3 ubiquitin ligase complex, in PHP at the Drosophila NMJ ${ }^{45}$, suggesting a key function of the UPS in both synaptic compartments during PHP at this synapse.

Trim32, the human ortholog of thin, is required for synaptic downscaling in cultured hippocampal rat neurons ${ }^{46}$, as well as long-term potentiation in hippocampal mouse slices ${ }^{47}$, implying a broader role of this E3 ubiquitin ligase in synaptic plasticity. Trim32 has been implicated in various neurological disorders, such as depression ${ }^{48}$, Alzheimer's Disease ${ }^{49}$, Autism Spectrum Disorder 48,50, or attention deficit hyperactivity disorder ${ }^{51}$. It will be exciting to explore potential links between Trim32-dependent control of synaptic homeostasis and these disorders in the future.

\section{METHODS}

\section{Fly stocks and genetics}

Drosophila stocks were maintained at $21^{\circ} \mathrm{C}-25^{\circ} \mathrm{C}$ on normal food. The $w^{1118}$ strain was used as the wild-type (WT) control. GluRIIASP16 mutants 4 and dysbindin ${ }^{1}$ mutants ${ }^{22}$ were a kind gift from Graeme Davis' lab. thin ${ }^{\Delta A}$ mutants and UAS-abba transgenic flies, now referred to as UAS-thin ${ }^{25}$, were a generous gift from Erika Geisbrecht. For pan-neuronal expression, the elav ${ }^{c 155}$ Gal4 (on the X chromosome) driver line was used and analysis was restricted to male larvae. For expression in muscle cells, the 24B-Gal4 driver line was used. Both driver lines were obtained from the Bloomington Drosophila Stock Center (Bloomington, IN, USA). Standard second and third chromosome balancer lines (Bloomington) and genetic strategies were used for all crosses and for maintaining the mutant lines. For the generation of transgenic flies carrying UAS-thin::mcherry, constructs based on the pUAST-attB vector backbone were injected into the ZP-attP-86Fb fly line harboring a landing site on the third chromosome according to standard procedures ${ }^{52}$.

\section{Cell culture and transfection}

Schneider S2 cells were cultivated in standard Schneider's Drosophila medium ( Gibco $^{\mathrm{TM}}$ ) containing 10\% fetal calf serum and 5\% Penicilin/Streptomycin at $25{ }^{\circ} \mathrm{C}$. For immunohistochemistry and microscopy cells, were plated on cover slips in 12 well plates with $80 \%$ density and transfected with $1.5 \mu \mathrm{g}$ (total) vector DNA using FuGENE® HD Transfection Reagent according to the standard protocol. Vectors used were: pMT-Gal4 (Addgene), pUAS-thin-mCherry and 
pUAS-venus-dysbindin (Dion Dickman). $24 \mathrm{~h}$ after plating, $\mathrm{CuSO}_{4}(0.5 \mathrm{mM})$ was added to the culture for $24 \mathrm{~h}$ to induce the expression of the pMT vector driving Gal4, which in turn drives transcription of UAS constructs.

\section{Plasmid construction}

All plasmids were generated by standard restriction enzyme ligation.

For the pUAS_attB_mCherry_thin vector, mCherry was cloned into pUAS_attB (Addgene) via EcoRI/Notl using the following primers

(fw: 5 ctcggcgcgccaATGGTGAGCAAGGGC GAGGAG-3',

rev: cgcggtaccttaCTTGTACAGCTCGTCCA TGCCGC-3').

thin was amplified from Drosophila CDNA by PCR using the following primers

(fw

CGGAATTCATGGAGCAATTCGAGCA GCTGTTGACG,

rev:

CGTCTAGAATGAAGACTTGGACGC

GGTGATTCTCTCG) and then cloned into the pUAS-attB-mcherry vector via Notl/Xbal.

Correct cloning was confirmed by sequencing of all final vectors.

\section{Electrophysiology}

Electrophysiological recordings were made from third-instar larvae at the wandering stage. Larvae were dissected and sharp-electrode recordings were made from muscle 6 in abdominal segments 3 and 4 using an Axoclamp 900A amplifier (Molecular Devices). The extracellular HL3 saline contained (in $\mathrm{mM}$ ): $70 \mathrm{NaCl}, 5 \mathrm{KCl}, 10$ $\mathrm{MgCl}_{2}, 10 \mathrm{Na}$-Hepes, 115 sucrose, 5 trehalose, 5 HEPES, $1.5 \mathrm{CaCl}_{2}$. To induce PHP, larvae were incubated with $20 \mu \mathrm{M}$ PhTX-433 (Cat \# sc-255421, Santa Cruz Biotechnology) for $10 \mathrm{~min}$ at room temperature after partial dissection (see ${ }^{5}$ ). AP-evoked EPSCs were induced by stimulating hemisegmental nerves with single APs (0.3 ms stimulus duration, $0.3 \mathrm{~Hz}$ ), and recorded with a combination of a HS-9A x10 and a HS-9A x0.1 headstage (Molecular Devices) in two-electrode voltage clamp (TEVC) mode. mEPSPs and $\mathrm{mEPSC}$ s were recorded with one or two HS-9A x0.1 headstage(s) (Molecular Devices), respectively. Muscle cells were clamped to a membrane potential of $-65 \mathrm{mV}$ for EPSCs and $-100 \mathrm{mV}$ for mEPSCs to increase the signal-to-noise ratio. A total of 50 EPSCs were averaged to obtain the mean EPSC amplitude for each NMJ. RRP size was calculated by the method of cumulative EPSC amplitudes 53. NMJs were stimulated with $60-\mathrm{Hz}$ trains (60 stimuli, 5 trains per cell), and the cumulative EPSC amplitude was obtained by back-extrapolating a linear fit to the last 15 cumulative EPSC amplitude values of the $60-\mathrm{Hz}$ train to time zero.

\section{Immunohistochemistry and microscopy}

Drosophila NMJ: Third-instar larval preparations were fixed for 3 min with Bouin's fixative $(100 \%$, Sigma-Aldrich, HT-10132) for confocal microscopy, or $100 \%$ ice-cold Ethanol for $10 \mathrm{~min}$ for STED microscopy. Preparations were washed thoroughly with PBS containing $0.1 \%$ Triton $X-100$. After washing, preparations were blocked with 3\% normal goat serum in PBS containing $0.1 \%$ Triton $X-100$. Incubation with the primary antibody was done at $4{ }^{\circ} \mathrm{C}$ on a rotating platform overnight.

The following antibodies and dilutions were used for NMJ stainings: (Primary) anti-Bruchpilot (nc82, mouse, DSHB, AB_2314866, 1:100), anti-GFP (rabbit, Thermo Fisher Scientific, G10362, 1:500), anti-GFP (mouse, Thermo Fisher Scientific, A-11120, 1:500), anti-DsRed (rabbit, Clontech, 
sc-390909, 1:500), anti-SYNORF1 (Synapsin, 3C11, mouse, DSHB, AB_528479, 1:250), anti-HRP AlexaFluor $647 \quad$ (goat, Jackson ImmunoResearch 123-605-021, 1:200). For confocal microscopy AlexaFluor anti-mouse 488 (Thermo Fisher Scientific; 1:500) and Alexa Fluor antiguinea pig 555 (Thermo Fisher Scientific; 1:400) were applied overnight at $4^{\circ} \mathrm{C}$ on a rotating platform. For gSTED microscopy (Figure 6,S3) the following secondary antibodies (1:100) were applied for $2 \mathrm{~h}$ at room temperature on a rotating platform: Atto 594 (anti-mouse, Sigma-Aldrich, 76085) and Abberior STAR 635 P (anti-rabbit, Abberior, 53399). Experimental groups of a given experiment were processed in parallel in the same tube. Preparations were mounted onto slides with ProLong Gold (Life Technologies, P36930).

S2 cell culture: $S 2$ cells grown on coverslips were washed with PBST (PBS $+0.1 \%$ TritonX-100) and fixed with $10 \%$ PFA for $10 \mathrm{~min}$. After washing three times with PBST, preparations were blocked with 5\% normal goat serum in PBST for 30 min. Incubation with primary antibody was done at RT on a rotating platform for $2 \mathrm{~h}$. The following antibodies were used for S2 cell stainings: anti-thin (guineapig, gift from Erika R. Geisbrecht, 1:200), antidysbindin (mouse, gift from Dion Dickman, 1:400). After washing three times with PBST, cells were incubated with secondary antibodies Alexa Fluor anti-guinea pig 555 and Alexa Fluor anti-mouse 488 (Thermo Fisher Scientific; $1: 400$ ) at RT on a rotating platform for $2 \mathrm{~h}$. Cover slips were mounted onto slides with ProLong Gold (Life Technologies, P36930) after three PBST washes.

Confocal and gSTED microscopy: Images were acquired with an inverse Leica TCS SP8 STED 3X microscope (Leica Microsystems, Germany) of the
University of Zurich Center for Microscopy and Image Analysis. Excitation light $(580 \mathrm{~nm}$ or $640 \mathrm{~nm})$ of a flexible white light laser was focused onto the specimen using a 100x objective (HC PL APO 1.40 NA Oil STED WHITE; Leica Microsystems, Germany) with immersion oil conforming to ISO 8036 with a diffraction index of $n=1.5180$ (Leica Microsystems, Germany). For gSTED imaging, the flexible white light laser was combined with a $775 \mathrm{~nm}$ STED depletion laser. Emitted light was detected with two HyD detectors in photon counting mode (Leica Microsystems, Germany). Pixel size was $20 \times 20 \mathrm{~nm}$ and z-stacks were acquired with a step size of $120 \mathrm{~nm}$. For STED imaging, we used time-gated single photon detection (empirical adjustment within a fluorescence lifetime interval from 0.7 to $6.0 \mathrm{~ns}$ ). Pixel size was $20 \times 20 \mathrm{~nm}$ and z-stacks were acquired with a step size of 120 or 130 $\mathrm{nm}$. Line accumulation was set to 1 and 6 for confocal and STED imaging, respectively. Images were acquired with LAS X software (Leica Application Suite $X$, version 2.0; Leica Microsystems, Germany). Experimental groups were imaged side-by-side with identical settings.

Images were processed and deconvolved with Huygens Professional (Huygens compute engine 17.04, Scientific Volume Imaging B.V., Netherlands). In brief, the "automatic background detection" tool (radius $=0.7 \mu \mathrm{m}$ ), and the "auto stabilize" feature were used to correct for background and lateral drift. Images were deconvolved using the Good's roughness Maximum Likelihood algorithm with default parameter settings (maximum iterations: 10; signal to noise ratio: 7 for STED and 15 for confocal; quality threshold: 0.003 ). 


\section{Western blot}

Transfected cells in 12-well-plates were washed with PBS and lysed by adding $50 \mu \mathrm{l}$ of RIPA buffer $(50 \mathrm{mM}$ Tris, $\mathrm{pH}$ 8.0, $150 \mathrm{mM} \mathrm{NaCl}, 1 \%$ Nonidet P-40, $0.5 \%$ deoxycholate, $0.1 \%$ SDS, $0.2 \mathrm{mM}$ sodium vanadate, $10 \mathrm{mM} \mathrm{NaF}, 0.4 \mathrm{mM}$ EDTA, $\quad 10 \%$ glycerol) containing protease inhibitors (cOmplete ${ }^{\mathrm{TM}}$, Mini, EDTA-free Protease Inhibitor Cocktail, Sigma) for $30 \mathrm{~min}$ on ice. The lysates were sonified three times for $1 \mathrm{~min}$ and boiled for $5 \mathrm{~min}$ in SDS-sample buffer containing $5 \% \quad$ ß-Mercaptoethanol. Samples were separated on acrylamide gels using SDS-PAGE, then transferred to nitrocellulose membranes (Amersham Hibond GE healthcare). After blocking in 5\% milk in PBST, membranes were incubated in the following primary antibodies: anti-GFP (rabbit, Thermo Fisher Scientific, G10362; 1:500), anti-DsRed (rabbit, Clontech, sc-390909, 1:500) in blocking solution overnight. Horseradish peroxidase-conjugated secondary Abs (anti-mouse-HRP and anti-rabbit-HRP 1:2000 in blocking solution) were applied to membranes for $2 \mathrm{~h}$. Detection was performed using ECL Reagent (GE Healthcare, Chicago, IL, USA).

\section{Data analysis}

Electrophysiology data were acquired with Clampex (Axon CNS, Molecular Devices) and analysed with customwritten routines in Igor Pro (Wavemetrics). For the genetic screen data, mEPSPs were detected with a template matching algorithm implemented in Neuromatic (Rothman \& Silver, 2018) running in Igor Pro (Wavemetrics). The average mEPSP amplitude was calculated from all detected events in a recording after visual inspection for false positives. For the rest of the data, mEPSC data were analysed using routines written with scientific python libraries, including numpy, scipy, IPython and neo ${ }^{54}$, and
mEPSCs were detected using an implementation of a template-matching algorithm ${ }^{55}$.

Microscopy images were analysed using custom-written routines in ImageJ (version 1.51n, National Institutes of Health, USA). Brp quantification was performed as follows: First, individual Brp puncta were isolated by segmenting binary fluorescence intensity threshold masks (15\% or $35 \%$ of the maximum intensity value) of background corrected (rolling ball, radius $=1 \mu \mathrm{m})$ and filtered $(3 \times 3$ median) maximum intensity projection images. The number of Brp objects in the mask served as a proxy for $A Z$ number, and was normalized to the area of the HRP mask (binary mask, $15 \%$ or $35 \%$ of the maximum intensity value). Average Brp-intensity values were calculated for each Brp punctum from background-corrected, unfiltered maximum intensity projection images.

Statistical analyses were done using RStudio Team (2021). RStudio: Integrated Development Environment for R. RStudio, PBC, Boston, MA. For more than two factors, we used two-way ANOVA followed by Tukey's post hoc test to correct for multiple comparisons between genotypes and conditions. For one factor with more than two groups, one-way ANOVA with Tukey's multiple comparisons was performed. Twosided Student's t-tests or nonparametric Mann-Whitney $U$ tests were used for comparison between two groups after a Shapiro-Wilk test and a Levene's test. Statistical significance was set to 0.05 $\left.{ }^{*}\right), 0.01\left(^{* *}\right)$ and $0.001\left(^{* * *}\right)$. Power analysis was performed using the pwrpackage of Rstudio to estimate the minimum sample size for a power above $\geq 0.8$ and a significance level of 0.05 for two-sided Student's t tests or MannWhithiney $U$ tests. Data are given as mean \pm s.e.m.

Figures were assembled using GIMP (The GIMP team, 2.8.10, 
www.gimp.org) and Inkscape (Inkscape project, 0.92.2. http://www.inkscape.org).

Acknowledgement: We are grateful to members of the Müller lab for helpful discussions and critical comments on the manuscript. We thank Dr. Damian Szklarczyk for help with STRING-based protein-protein interaction analysis used for prioritization of E3 ligases.

Funding: This research was funded a Swiss National Science Foundation Assistant Professor grant (PP00P315), and an European Research Council Starting grant (SynDegrade-679881) to MM.

Author Contributions: $\mathrm{MB}-\mathrm{C}, \mathrm{KS}$ and MM conceptualized and designed experiments. MB-C and $\mathrm{KS}$ conducted research and analysed data. MB-C, KS and MM interpreted data. MM and MBC wrote the manuscript.

Competing Interests: The authors declare no competing interests.

Data Availability: All data needed to evaluate the conclusions in the paper are present in the paper and the Supplementary Materials and are available upon reasonable request.

\section{REFERENCES}

1. Turrigiano, G. G. The Self-Tuning Neuron: Synaptic Scaling of Excitatory Synapses. Cell 135, 422-435 (2008).

2. Delvendahl, I. \& Müller, M. Homeostatic plasticity-a presynaptic perspective. Current Opinion in Neurobiology 54, 155162 (2019).

3. Marder, E. \& Goaillard, J.-M. Variability, compensation and homeostasis in neuron and network function. 7, 563-574
(2006).

4. Petersen, S. A., Fetter, R. D., Noordermeer, J. N., Goodman, C. S. \& DiAntonio, A. Genetic analysis of glutamate receptors in Drosophila reveals a retrograde signal regulating presynaptic transmitter release. Neuron 19, 1237-1248 (1997).

5. Frank, C. A., Kennedy, M. J., Goold, C. P., Marek, K. W. \& Davis, G. W. Mechanisms underlying the rapid induction and sustained expression of synaptic homeostasis. Neuron 52, 663-677 (2006).

6. Turrigiano, G. G., Leslie, K. R., Desai, N. S., Rutherford, L. C. \& Nelson, S. B. Activity-dependent scaling of quantal amplitude in neocortical neurons. Nature 2012 486:7401 391, 892-896 (1998).

7. Mullins, C., Fishell, G. \& Tsien, R. W. Unifying Views of Autism Spectrum Disorders: A Consideration of Autoregulatory Feedback Loops. Neuron 89, 1131-1156 (2016).

8. Dickman, D. \& Wondolowski, J. Emerging links between homeostatic synaptic plasticity and neurological disease. Front. Cell. Neurosci. 7, (2013).

9. Orr, B. O. et al. Presynaptic Homeostasis Opposes Disease Progression in Mouse Models of ALS-Like Degeneration: Evidence for Homeostatic Neuroprotection. Neuron 107, 95-111.e6 (2020).

10. Perry, S., Han, Y., Das, A. \& Dickman, D. Homeostatic plasticity can be induced and expressed to restore synaptic strength at neuromuscular junctions undergoing ALS-related degeneration. Human Molecular Genetics 26, 4153-4167 (2017).

11. Cohen, L. D. et al. Metabolic turnover of synaptic proteins: kinetics, interdependencies and implications for synaptic 
maintenance. PLoS ONE 8, e63191 (2013).

12. Zheng, N. \& Shabek, N. Ubiquitin Ligases: Structure, Function, and Regulation.

https://doi.org/10.1146/annurevbiochem-060815-014922 (2017).

doi:10.1146/annurev-biochem060815-014922

13. George, A. J., Hoffiz, Y. C., Charles, A. J., Zhu, Y. \& Mabb, A. M. A Comprehensive Atlas of E3 Ubiquitin Ligase Mutations in Neurological Disorders. Front. Genet. 9, (2018).

14. Hegde, A. N. The ubiquitinproteasome pathway and synaptic plasticity. Learn. Mem. 17, 314327 (2010).

15. Yao, I. et al. SCRAPPERdependent ubiquitination of active zone protein RIM1 regulates synaptic vesicle release. Cell 130, 943-957 (2007).

16. Russo, A. et al. The E3 ligase Highwire promotes synaptic transmission by targeting the NADsynthesizing enzyme dNmnat. EMBO reports 20, (2019).

17. Ramirez, J. et al. The ubiquitin ligase Ariadne-1 regulates neurotransmitter release via ubiquitination of NSF. J. Biol. Chem. 100408 (2021). doi:10.1016/j.jbc.2021.100408

18. Wang, X., Wang, Q., Engisch, K. L. \& Rich, M. M. Activity-dependent regulation of the binomial parameters $p$ and $n$ at the mouse neuromuscular junction in vivo. Journal of Neurophysiology 104, 2352-2358 (2010).

19. Plomp, J. J., van Kempen, G. T. \& Molenaar, P. C. Adaptation of quantal content to decreased postsynaptic sensitivity at single endplates in alpha-bungarotoxintreated rats. J. Physiol. (Lond.) 458, 487-499 (1992).

20. Cull-Candy, S. G., Miledi, R.,
Trautmann, A. \& Uchitel, O. D. On the release of transmitter at normal, myasthenia gravis and myasthenic syndrome affected human end-plates. J. Physiol. (Lond.) 299, 621-638 (1980).

21. Delvendahl, I., Kita, K. \& Müller, M. Rapid and sustained homeostatic control of presynaptic exocytosis at a central synapse. Proc. Natl. Acad. Sci. U.S.A. 116, 2378323789 (2019).

22. Dickman, D. K. \& Davis, G. W. The Schizophrenia Susceptibility Gene dysbindin Controls Synaptic Homeostasis. Science 326, 11271130 (2009).

23. Müller, M., Pym, E. C. G., Tong, A. \& Davis, G. W. Rab3-GAP Controls the Progression of Synaptic Homeostasis at a Late Stage of Vesicle Release. Neuron 69, 749762 (2011).

24. Wentzel, C., Delvendahl, I., Sydlik, S., Georgiev, O. \& Müller, M. Dysbindin links presynaptic proteasome function to homeostatic recruitment of low release probability vesicles. Nat Commun 9, 267 (2018).

25. LaBeau-DiMenna, E. M. et al. Thin, a Trim32 ortholog, is essential for myofibril stability and is required for the integrity of the costamere in Drosophila. Proc. Natl. Acad. Sci. U.S.A. 109, 17983-17988 (2012).

26. Domsch, K., Ezzeddine, N. \& Nguyen, H. T. Abba is an essential TRIM/RBCC protein to maintain the integrity of sarcomeric cytoarchitecture. J Cell Sci 126, 3314-3323 (2013).

27. Du, J. et al. In Vivo RNAi Screen Reveals Neddylation Genes as Novel Regulators of Hedgehog Signaling. PLOS ONE 6, e24168 (2011).

28. Ketosugbo, K. F., Bushnell, H. L. \& Johnson, R. I. A screen for E3 ubiquitination ligases that 
genetically interact with the adaptor protein Cindr during Drosophila eye patterning. PLOS ONE 12, e0187571 (2017).

29. Weyhersmüller, A., Hallermann, S., Wagner, N. \& Eilers, J. Rapid active zone remodeling during synaptic plasticity. J. Neurosci. 31, 6041-6052 (2011).

30. Müller, M., Liu, K. S. Y., Sigrist, S. J. \& Davis, G. W. RIM Controls Homeostatic Plasticity through Modulation of the ReadilyReleasable Vesicle Pool. J. Neurosci. 32, 16574-16585 (2012).

31. Jan, L. Y. \& Jan, Y. N. Antibodies to horseradish peroxidase as specific neuronal markers in Drosophila and in grasshopper embryos. PNAS 79, 2700-2704 (1982).

32. Kittel, R. J. et al. Bruchpilot promotes active zone assembly, $\mathrm{Ca} 2+$ channel clustering, and vesicle release. Science 312, 1051-1054 (2006).

33. Locke, M., Tinsley, C. L., Benson, M. A. \& Blake, D. J. TRIM32 is an E3 ubiquitin ligase for dysbindin. Human Molecular Genetics 18, 2344-2358 (2009).

34. Wan, H. I. et al. Highwire regulates synaptic growth in Drosophila. Neuron 26, 313-329 (2000).

35. van Roessel, P., Elliott, D. A., Robinson, I. M., Prokop, A. \& Brand, A. H. Independent regulation of synaptic size and activity by the anaphase-promoting complex. Cell 119, 707-718 (2004).

36. DiAntonio, A. et al. Ubiquitinationdependent mechanisms regulate synaptic growth and function. Nature 2012 486:7401 412, 449452 (2001).

37. Kudryashova, E., Kudryashov, D., Kramerova, I. \& Spencer, M. J. Trim32 is a ubiquitin ligase mutated in limb girdle muscular dystrophy type $2 \mathrm{H}$ that binds to skeletal muscle myosin and ubiquitinates actin. $\mathrm{J} \mathrm{Mol} \mathrm{Biol} \mathrm{354,}$ 413-424 (2005).

38. Cijsouw, T. et al. Mapping the Proteome of the Synaptic Cleft through Proximity Labeling Reveals New Cleft Proteins. Proteomes 6, 48 (2018).

39. Chen, K., Richlitzki, A., Featherstone, D. E., Schwärzel, M. \& Richmond, J. E. Tomosyndependent regulation of synaptic transmission is required for a late phase of associative odor memory. Proc. Natl. Acad. Sci. U.S.A. 108, 18482-18487 (2011).

40. Hatsuzawa, K., Lang, T., Fasshauer, D., Bruns, D. \& Jahn, $R$. The R-SNARE motif of tomosyn forms SNARE core complexes with syntaxin 1 and SNAP-25 and down-regulates exocytosis. J. Biol. Chem. 278, 31159-31166 (2003).

41. Pilgram, G. S. K., Potikanond, S., van der Plas, M. C., Fradkin, L. G. \& Noordermeer, J. N. The RhoGAP crossveinless-c interacts with Dystrophin and is required for synaptic homeostasis at the Drosophila neuromuscular junction. J. Neurosci. 31, 492-500 (2011).

42. Kreko-Pierce, T. \& Eaton, B. A. The Drosophila LC8 homolog cut up specifies the axonal transport of proteasomes. J Cell Sci 130, 3388-3398 (2017).

43. Speese, S. D., Trotta, N., Rodesch, C. K., Aravamudan, B. \& Broadie, $\mathrm{K}$. The ubiquitin proteasome system acutely regulates presynaptic protein turnover and synaptic efficacy. Current Biology 13, 899-910 (2003).

44. Dickman, D. K., Tong, A. \& Davis, G. W. Snapin is Critical for Presynaptic Homeostatic Plasticity. J. Neurosci. 32, 8716-8724 (2012).

45. Kikuma, K. et al. Cul3 and 
insomniac are required for rapid ubiquitination of postsynaptic targets and retrograde homeostatic signaling. Nat Commun 10, 2998 (2019).

46. Srinivasan, B., Samaddar, S., Mylavarapu, S. V. S., Clement, J. P. \& Banerjee, S. Homeostatic scaling is driven by a translationdependent degradation axis that recruits miRISC remodelling. bioRxiv 2020.04.01.020164 (2020).

47. Ntim, M. et al. TRIM32 Deficiency Impairs Synaptic Plasticity by Excitatory-Inhibitory Imbalance via Notch Pathway. Cereb Cortex 30, 4617-4632 (2020).

48. Ruan, C.-S. et al. Deletion of TRIM32 protects mice from anxiety- and depression-like behaviors under mild stress. European Journal of Neuroscience 40, 2680-2690 (2014).

49. Yokota, T. et al. Brain site-specific gene expression analysis in Alzheimer's disease patients. Eur $J$ Clin Invest 36, 820-830 (2006).

50. Lionel, A. C. et al. Disruption of the ASTN2/TRIM32 locus at 9q33.1 is a risk factor in males for autism spectrum disorders, ADHD and other neurodevelopmental phenotypes. Human Molecular Genetics 23, 2752-2768 (2014).

51. Lionel, A. C. et al. Rare copy number variation discovery and cross-disorder comparisons identify risk genes for ADHD. Sci Transl Med 3, 95ra75-95ra75 (2011).

52. Bischof, J., Sheils, E. M., Björklund, M. \& Basler, K. Generation of a transgenic ORFeome library in Drosophila. Nat Protoc 9, 1607-1620 (2014).

53. Schneggenburger, R., Meyer, A. C. \& Neher, E. Released fraction and total size of a pool of immediately available transmitter quanta at a calyx synapse. Neuron 23, 399-
409 (1999).

54. Garcia, S. et al. Neo: an object model for handling electrophysiology data in multiple formats. Front. Neuroinform. 8, (2014).

55. Clement, J.D., Bekkers, J.M. Detection of spontaneous synaptic events with an optimally scaled template. Biophysical Journal 73, 220-229 (1997).

56. Larkin, A., Marygold, S. J., Acids, G. A. N.2021. FlyBase: updates to the Drosophila melanogaster knowledge base. academic.oup.com doi:10.1093/nar/gkaa1026

57. Li, W. et al. Genome-wide and functional annotation of human E3 ubiquitin ligases identifies MULAN, a mitochondrial E3 that regulates the organelle's dynamics and signaling. PLOS ONE 3, e1487 (2008).

58. Hu, Y. et al. An integrative approach to ortholog prediction for disease-focused and other functional studies. BMC Bioinformatics 12, 357-16 (2011).

59. Celniker, S. E. et al. Unlocking the secrets of the genome. Nature 2012 486:7401 459, 927-930 (2009).

60. Chintapalli, V. R., Wang, J. \& Dow, J. A. T. Using FlyAtlas to identify better Drosophila melanogaster models of human disease. Nat. Genet. 39, 715-720 (2007).

61. Mering, von, C. et al. STRING: known and predicted proteinprotein associations, integrated and transferred across organisms. Nucleic Acids Res. 33, D433-7 (2005).

62. Pazos Obregón, F., Papalardo, C., Castro, S., Guerberoff, G. \& Cantera, R. Putative synaptic genes defined from a Drosophila whole body developmental transcriptome by a machine 
bioRxiv preprint doi: https://doi.org/10.1101/2021.06.16.448554; this version posted June 16, 2021. The copyright holder for this preprint (which was not certified by peer review) is the author/funder, who has granted bioRxiv a license to display the preprint in perpetuity. It is made available under aCC-BY-NC-ND 4.0 International license.

learning approach. BMC Genomics

16, 694 (2015).

63. Pazos Obregón, F. et al. An improved catalogue of putative

synaptic genes defined exclusively by temporal transcription profiles through an ensemble machine learning approach. BMC Genomics 20, 1011-8 (2019). 